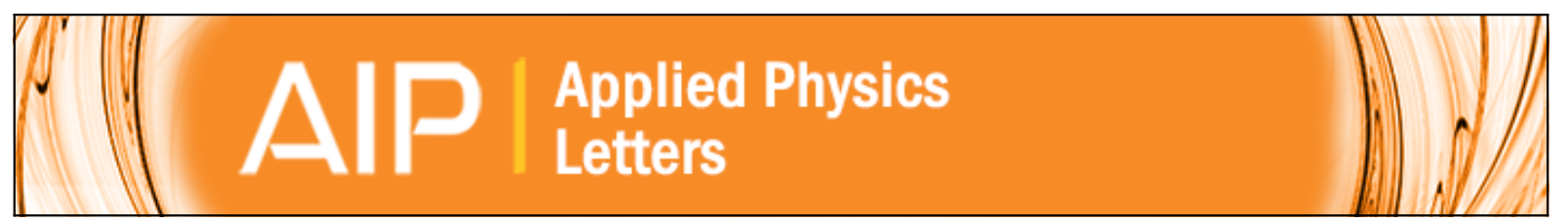

\title{
The time resolved measurement of ultrashort terahertz-band electric fields without an ultrashort probe
}

D. A. Walsh, E. W. Snedden, and S. P. Jamison

Citation: Applied Physics Letters 106, 181109 (2015); doi: 10.1063/1.4919899

View online: http://dx.doi.org/10.1063/1.4919899

View Table of Contents: http://scitation.aip.org/content/aip/journal/apl/106/18?ver=pdfcov

Published by the AIP Publishing

\section{Articles you may be interested in}

A quasi-optical vector near-field measurement system at terahertz band

Rev. Sci. Instrum. 85, 064702 (2014); 10.1063/1.4882321

Time-resolved measurement of pulse-to-pulse heating effects in a terahertz quantum cascade laser using an $\mathrm{NbN}$ superconducting detector

Appl. Phys. Lett. 103, 061120 (2013); 10.1063/1.4818584

Single-shot measurement of a terahertz electric-field waveform using a reflective echelon mirror

Appl. Phys. Lett. 103, 051103 (2013); 10.1063/1.4817011

Optical phase detection in a 4-N,N-dimethylamino- $4^{\prime}-\mathrm{N}^{\prime}$-methyl-stilbazolium tosylate crystal for terahertz time domain spectroscopy system at $1.55 \mu \mathrm{m}$ wavelength

Appl. Phys. Lett. 97, 111112 (2010); 10.1063/1.3490706

A study of background signals in terahertz apertureless near-field microscopy and their use for scattering-probe imaging

J. Appl. Phys. 105, 113117 (2009); 10.1063/1.3141727
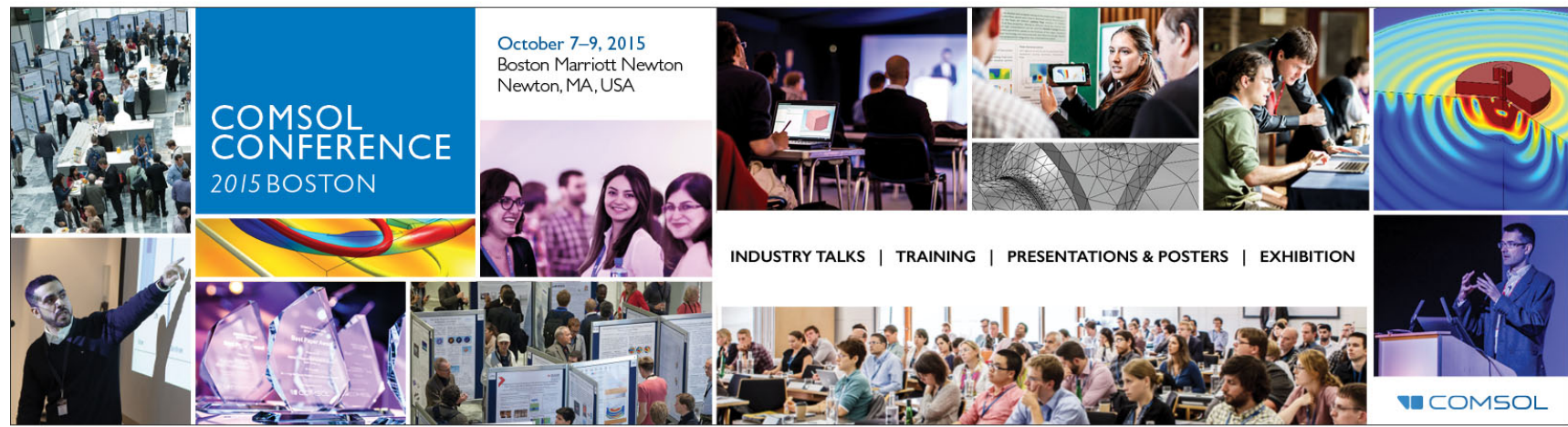


\title{
The time resolved measurement of ultrashort terahertz-band electric fields without an ultrashort probe
}

\author{
D. A. Walsh, ${ }^{1, a)}$ E. W. Snedden, ${ }^{1}$ and S. P. Jamison ${ }^{1,2}$ \\ ${ }^{1}$ Accelerator Science and Technology Centre, STFC Daresbury National Laboratory, Warrington WA4 4AD, \\ United Kingdom \\ ${ }^{2}$ Photon Science Institute, The University of Manchester, Manchester M13 9PL, United Kingdom
}

(Received 13 February 2015; accepted 27 April 2015; published online 7 May 2015)

\begin{abstract}
The time-resolved detection of ultrashort pulsed THz-band electric field temporal profiles without an ultrashort laser probe is demonstrated. A non-linear interaction between a narrow-bandwidth optical probe and the $\mathrm{THz}$ pulse transposes the $\mathrm{THz}$ spectral intensity and phase information to the optical region, thereby generating an optical pulse whose temporal electric field envelope replicates the temporal profile of the real $\mathrm{THz}$ electric field. This optical envelope is characterised via an autocorrelation based FROG (frequency resolved optical gating) measurement, hence revealing the $\mathrm{THz}$ temporal profile. The combination of a narrow-bandwidth, long duration, optical probe, and self-referenced FROG makes the technique inherently immune to timing jitter between the optical probe and $\mathrm{THz}$ pulse and may find particular application where the $\mathrm{THz}$ field is not initially generated via ultrashort laser methods, such as the measurement of longitudinal electron bunch profiles in particle accelerators. (C) 2015 AIP Publishing LLC. [http://dx.doi.org/10.1063/1.4919899]
\end{abstract}

The characterisation of ultrashort pulse $\mathrm{THz}$ radiation has commonly proceeded through the electro-optic sampling (EOS) of the THz field with a synchronised optical probe in an electro-optically responsive material, either through scanning measurements such as $\mathrm{THz}$ time domain spectrometry (THz-TDS $)^{1-3}$ or through single shot arrangements such as spatial encoding ${ }^{4,5}$ and spectral decoding, ${ }^{6-11}$ or their subsequent improvements. ${ }^{12,13}$ These measurements run into difficulty when a sufficiently broad bandwidth ultrashort pulse probe is not available, thus limiting temporal resolution of the retrieved field and preventing an accurate measurement. Additionally, jitter between the probe and the $\mathrm{THz}$ pulse can also blur the profile (scanning methods) or move it outside the sampling window altogether (single shot methods). Whilst such jitter is not typically a problem for laser based $\mathrm{THz}$ sources, it can be a significant issue when the $\mathrm{THz}$ field is not laser derived. For example, in the diagnostic measurement of relativistic particle bunch longitudinal profiles in accelerators, the jitter can be high ( $>$ a few picoseconds) and the resolution requirements increasingly demanding $(<50$ fs). Such a diagnostic system must also be highly robust and reliable - not a particularly compatible requirement with the few tens of femtosecond duration ultrashort pulse lasers that would be required for scanning ${ }^{14,15}$ or single-shot methods. ${ }^{8}$

The problem of requiring a suitably broadband and synchronised probe was also historically encountered in the characterisation of ultrashort optical radiation, but the development of combined time- and frequency-domain methods such as FROG (frequency resolved optical gating) ${ }^{16-18}$ and SPIDER (spectral phase interferometry for direct electric-field reconstruction ${ }^{19}$ has provided the means to recover the temporal envelope, and phase evolution, of such pulses. These methods allow a copy of the pulse being measured to act as its own probe, and they can be configured to characterise a pulse in a

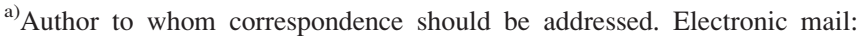
david.walsh@stfc.ac.uk
}

single shot. When combined these features remove any need for distinct, synchronised, ultrashort "probe" pulses at all. Unfortunately, there is an absence of such self-referenced techniques at $\mathrm{THz}$ frequencies, which can be attributed in part to the lack of suitably high pulse energies and compatible nonlinear materials. More fundamentally, in THz-TDS applications, the carrier envelope phase (CEP), which is not retrieved in standard FROG methods, is an essential part of the electric field that needs to be characterised. While this CEP problem has recently been overcome, in principle, by "ReD-FROG," 20 in this work, the FROG CEP ambiguity is bypassed through conversion of the full $\mathrm{THz}$ field, including the "carrier" and the envelope, into an optical envelope alone.

In this letter, we report the time resolved electro-optic measurement of $0-3.0 \mathrm{THz}$ electric field waveforms using a narrow-bandwidth quasi-continuous wave (CW) optical probe in conjunction with a FROG characterisation of the electro-optically modified probe. The temporal resolution of the electric field measurement is no longer derived from the duration of the probe and represents a method for achieving high temporal resolution measurements without the need for ultrashort lasers. The concept is demonstrated through a benchmarked proof-of-principle experiment involving the $\chi^{(2)}$ interaction between optical probe and THz waveforms in $\mathrm{ZnTe}$. By using a narrow bandwidth optical probe, the $\mathrm{THz}$ spectral information is remapped into up-converted optical waves, thereby reproducing the $\mathrm{THz}$ waveform in the envelope of an optical pulse. This optical envelope (三 spectral amplitude and phase) is then characterised through selfreferenced FROG allowing the THz pulse to be recovered.

The principle reported here allows for sub-100 fs resolution in $\mathrm{THz}$ waveform measurements with nanosecond or $\mathrm{CW}$ optical probes. For our proof-of-concept demonstration, a 10 ps duration "quasi-CW" probe is generated through 4-f spectral filtering of a $50 \mathrm{fs}$ duration pulse. This allows the $\mathrm{THz}$ pulse to be independently characterised with conventional 
electro-optic sampling with $50 \mathrm{fs}$ pulses and for the $\mathrm{THz}$ pulse to be conveniently generated from the same laser.

The electro-optic (EO) effect can be generally described as the sum- and difference-frequency generation between the frequency components of the optical probe and $\mathrm{THz}$ pulses. ${ }^{21,22}$ For the commonly used EO materials ZnTe and $\mathrm{GaP}$, the largest nonlinear effect is produced in (110) cut crystals where the optical and $\mathrm{THz}$ fields are polarised parallel to $[1 \overline{1} 0]$. The principal axes of the interaction in this system are then at $\pm 45^{\circ}$ to this polarisation. ${ }^{23-25}$ Using the result of Jamison et al., the wave generated by the EO interaction in the 2 principle axes ( $i=1$ or 2$)$ of the material in the time domain is expressed as

$$
E_{i}(t ; \tau) \propto \beta_{i} \frac{d}{d t}\left[E^{o p t}(t) \cdot E_{e f f}^{T H z}(t-\tau)\right],
$$

where $E^{o p t}(t)$ denotes the input optical field, $E_{\text {eff }}^{T H z}(t)$ is the effective input field including the effects of dispersion in $\chi^{(2)}$ and phase matching, and $\tau$ is the temporal delay between the optical and $\mathrm{THz}$ pulses. For the geometry specified above, the axis dependent constant $\beta_{i}$ takes the value of +1 or -1 depending on the axis, $i=1$ or 2 , being examined. As such, the newly generated waves can be separated from the input via a polariser orthogonal to the input optical polarisation. With dispersion data at $\mathrm{THz}$ frequencies available for common media determination of $E^{T H z}(t)$ follows from knowledge of $E_{\text {eff }}^{T H z}(t){ }^{22,26}$

If a narrow bandwidth continuous wave or long pulse (i.e., one that does not change significantly on the timescale of the $\mathrm{THz}$ field) is used for the probe field and the optical probe field oscillation is much faster than any temporal variations in the $\mathrm{THz}$ pulse, then this optical carrier frequency dominates the derivative, whereas the optical envelope is constant. This results in the mapping of the $\mathrm{THz}$ profile into a new pulse envelope after the crossed polariser geometry according to

$$
E(t ; \tau) \propto 2\left(\frac{d}{d t} E^{o p t}(t)\right) \cdot E_{e f f}^{T H z}(t) .
$$

This process is analogous to musical transposition, where a piece of music is changed from one key to another: the notes (spectral amplitudes) are shifted by a fixed frequency and the relative note timings (spectral phases) are kept the same, thereby preserving the melody (temporal profile). Due to this similarity, we call the process EO Transposition (EOT). We note that at $\mathrm{THz}$ frequencies, the absolute phase is essential to the reproduction of the pulse profile, but once shifted to the optical regime the absolute phase only affects the optical carrier wave; the optical envelope, and so the $\mathrm{THz}$ temporal profile, can be faithfully retrieved via standard FROG techniques thereby bypassing the CEP ambiguity.

An experimental validation of the EOT method was performed using the setup outlined in Figure 1. The THz source was a large area $(75 \mathrm{~mm} \times 75 \mathrm{~mm})$, lt-GaAs, photo-conductive antenna (PCA) with a horizontally applied bias of $\sim 100 \mathrm{kV}$. This was pumped with $90 \%$ of the output of a Ti:sapphire regenerative amplifier $(500 \mathrm{~Hz}, \sim 1.5 \mathrm{~mJ}, 50 \mathrm{fs}$ transform-limited pulse duration). The remaining $10 \%$ input pulse energy was propagated through a scanning delay line and a zero-dispersion 4-f spectral filter to form the optical probe. The bandwidth of the optical probe could then be varied using slit placed in the Fourier plane of the 4-f filter.

Both $\mathrm{THz}$ and optical probe were combined coaxially with the THz pulse using an ITO coated glass substrate and directed on to a $4 \mathrm{~mm} \mathrm{ZnTe} \mathrm{crystal.} \mathrm{A} \mathrm{flip} \mathrm{mirror} \mathrm{was} \mathrm{used} \mathrm{to}$ direct the ZnTe output to either a balanced detection set-up for EOS or to a crossed polarisation analyser for EOT. A quarter wave plate after the ZnTe was implemented differently in each scheme: circularising the polarisation of the transmitted light in balanced detection, and correcting for any impurity/ stress-induced residual birefringence in the $\mathrm{ZnTe}$ in EOT.

Measurement in the balanced detection scheme was performed using lock-in amplifier techniques referenced to the $250 \mathrm{~Hz}$ pulsing of the PCA bias voltage; the resulting time domain sampling of the $\mathrm{THz}$ waveform, using a $50 \mathrm{fs}$ probe pulse, is shown in Figure 3. For EOT measurements, the slit in

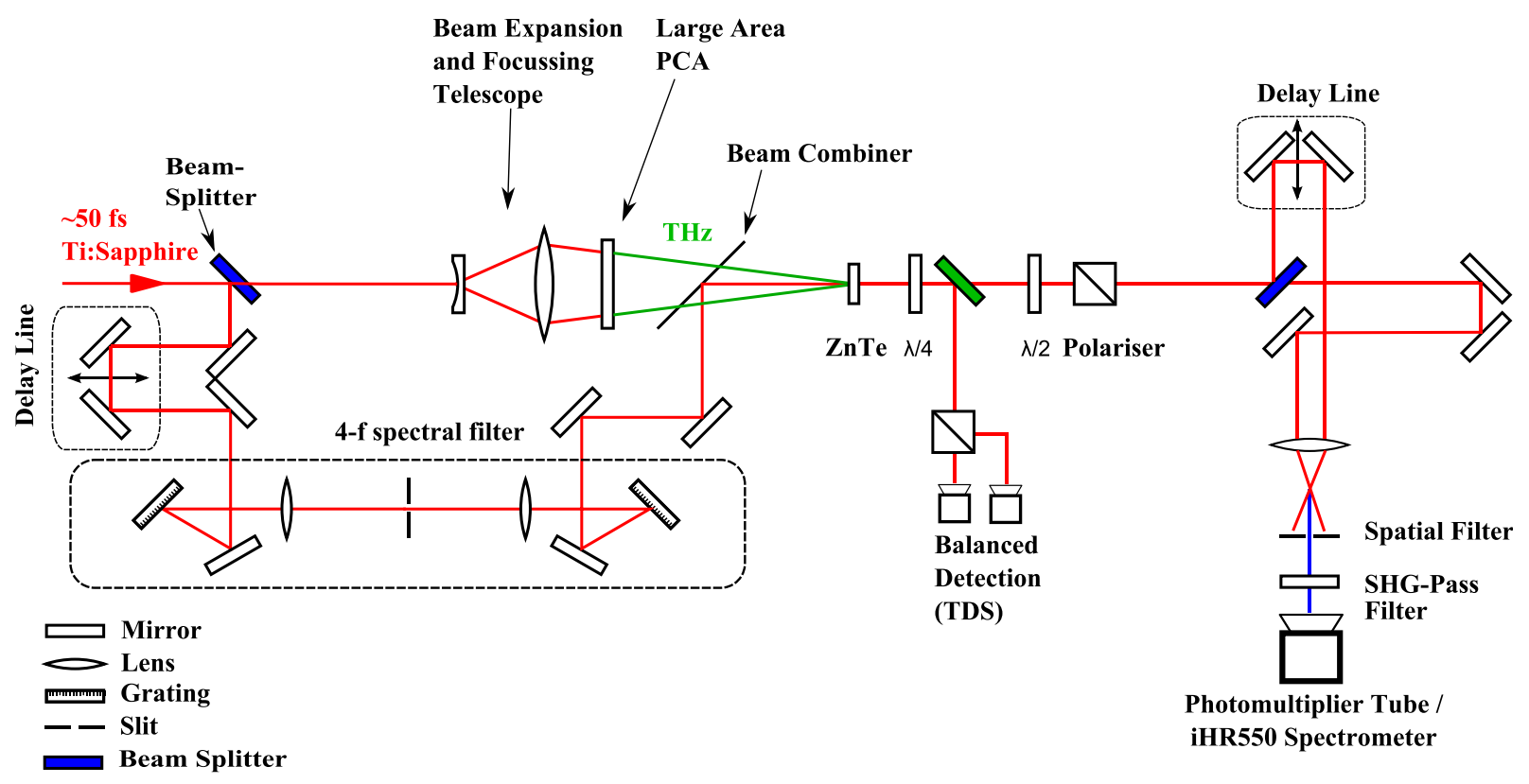

FIG. 1. Experimental layout for the demonstration of EOT-FROG. 
the 4-f filter was set to produce probe pulses $10 \mathrm{ps}$ in duration of which a small amount was leaked through the crossed polariser geometry and coupled into a bespoke non-collinear auto correlator based on $100 \mu \mathrm{m}$ thick barium borate cut for second harmonic generation of $800 \mathrm{~nm}$ light. This allowed confirmation of the probe duration, and the signal from this was aligned into an iHR550 imaging spectrometer coupled to a DiCAM pro intensified CCD camera, completing the FROG arrangement. After alignment, the probe was extinguished again in the crossed polarised arrangement to facilitate measurement of the transposed pulses alone. Due to the low energy that was available in the transposed pulse $(<0.5 \mathrm{~nJ})$, a multiple shot averaging of the intensified camera images was required to measure the SHG (second harmonic generation) spectrum at each delay point. This involved 256 frame integrations on the iCCD before readout and then summing 30 of these samples. The complete FROG spectrograms were recorded over 128 points with a step size of $62.5 \mathrm{fs}$.

The FROG spectrogram was analysed using Femtosoft Technologies commercial FROG retrieval code "FROG3," including image pre-processing for noise and background.

(a)

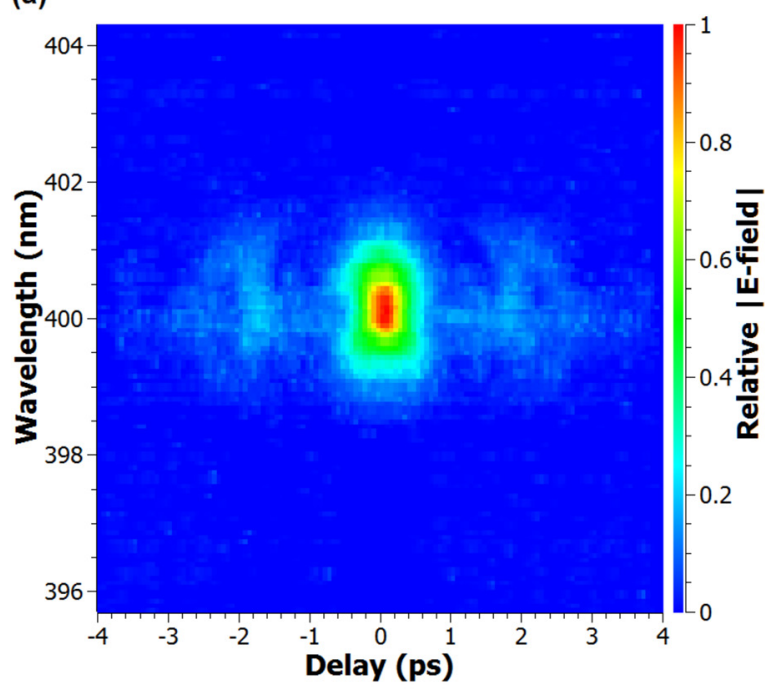

(b)

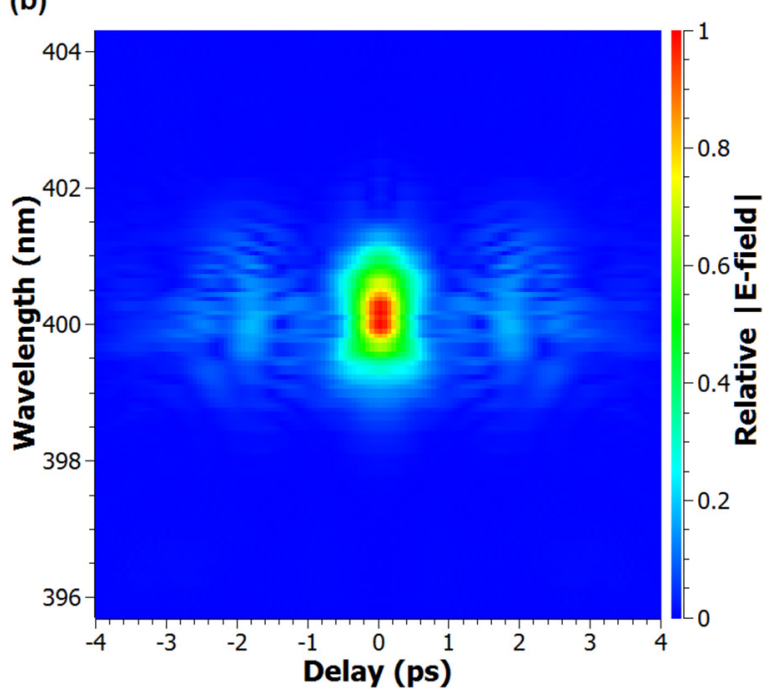

FIG. 2. (a) The measured spectrogram after noise reduction and (b) the recovered spectrogram. Both are displayed in false colour and proportional to the electric field envelope magnitude to enhance visibility.
The (noise-filtered) measured spectrogram is shown alongside the recovered spectrogram in Figure 2. The recovered spectrogram is in good agreement with the measured one, despite the remaining noise, yielding a FROG error of 0.007 for the $128 \times 128$ spectrogram.

The temporal intensity envelope and phase of the EOS and recovered EOT signals are compared in Figure 3. Despite the high level of noise in the measured spectrogram, it is quite clear that the temporal profile has been recovered quite faithfully.

For the data set presented here, the limited signal to noise together with the well known SHG FROG ambiguity for well-separated pulses, led to the trailing oscillation at 2 ps having a phase ambiguity of $\pm n \pi$, where $n$ is an integer. In this case, we have manually subtracted $\pi$ in order for the sign of the recovered $\mathrm{THz}$ field to match. Note that the polarity shift occurring within a "continuous" pulse at 0.5 ps was correctly retrieved from the FROG recovered phase. It is envisaged that in many situations, a higher signal to noise ratio would mitigate this ambiguity as the interaction at intermediate delays between the pulses would be measurable.

In summary, we have reported an experimental scheme that will enable the time resolved measurement of ultrashort $\mathrm{THz}$ radiation without the need for an ultrashort optical probe pulse of duration much less than the desired time resolution. This demonstration used 10 ps long probe pulses, but the

(a)

\section{Intensity}

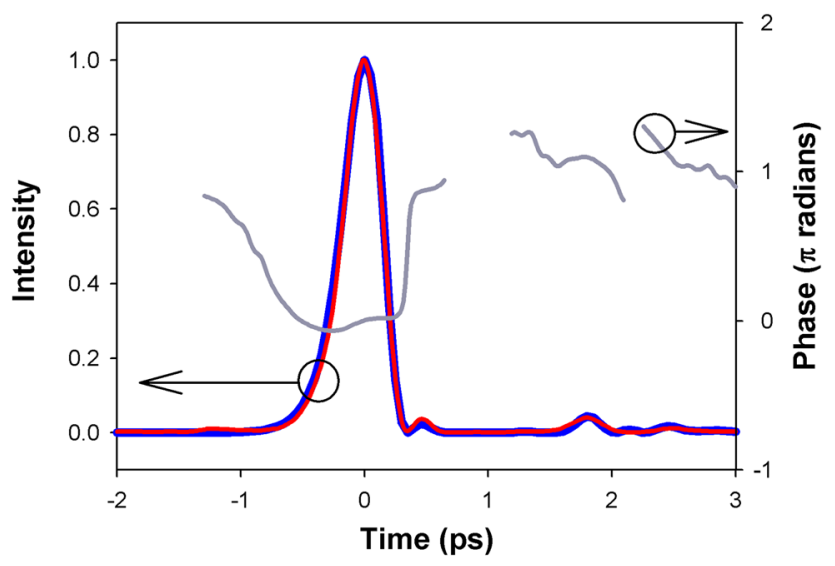

(b)

\section{Electric-Field}

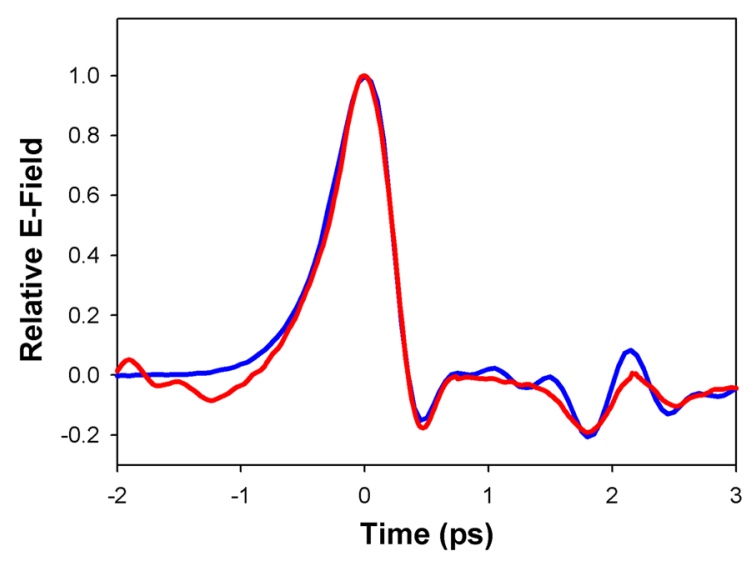

FIG. 3. (a) The $\mid$ E-field $\left.\right|^{2}$ of the THz-TDS trace in blue overlaid with the FROG-recovered intensity envelope in red. The grey dashed line represents the FROG-retrieved relative phase. (b) The E-field recovered via THz-TDS. 
principle is easily extended to the use of nanosecond long pulses for which the jitter tolerance between the $\mathrm{THz}$ pulse and that of the laser increases by a further two orders of magnitude. The only constraints on the EOT method are that the two pulses temporally overlap, and that any temporal variation in the $\mathrm{THz}$ and optical envelopes can be considered negligible compared to the optical carrier frequency. There remains the practical issue of having sufficient pulse energy to perform the FROG measurement, but these may be overcome via the implementation of a phase preserving amplification technique for the EOT pulse, such as optical parametric chirped pulse amplification, ${ }^{27}$ and/or a more sensitive FROG arrangement such as GRENOUILLE. ${ }^{28}$ Moving to GRENOUILLE will also enable single shot measurements of the temporal profile to be made with higher resolution than is possible via other single shot methods, being limited by the EO material response rather than the probe pulse properties.

${ }^{1}$ Q. Wu and X. C. Zhang, Appl. Phys. Lett. 67(24), 3523-3525 (1995).

${ }^{2}$ A. Nahata, A. S. Weling, and T. F. Heinz, Appl. Phys. Lett. 69(16), 2321-2323 (1996).

${ }^{3}$ A. Tomasino, A. Parisi, S. Stivala, P. Livreri, A. C. Cino, A. C. Busacca, M. Peccianti, and R. Morandotti, Sci. Rep. 3, 3116 (2013).

${ }^{4}$ J. Shan, A. S. Weling, E. Knoesel, L. Bartels, M. Bonn, A. Nahata, G. A. Reider, and T. F. Heinz, Opt. Lett. 25(6), 426-428 (2000).

${ }^{5}$ Y. Kawada, T. Yasuda, H. Takahashi, and S.-i. Aoshima, Opt. Lett. 33(2), 180-182 (2008).

${ }^{6}$ A. Galvanauskas, J. Tellefsen, Jr., A. Krotkus, M. Öberg, and B. Broberg, Appl. Phys. Lett. 60(2), 145-147 (1992).

${ }^{7}$ Z. Jiang and X.-C. Zhang, Appl. Phys. Lett. 72(16), 1945-1947 (1998).

${ }^{8}$ U. Schmidhammer, V. De Waele, J. R. Marquès, N. Bourgeois, and M. Mostafavi, Appl. Phys. B 94(1), 95-101 (2009).
${ }^{9}$ S. Jamison, G. Berden, W. Gillespie, P. Phillips, and A. MacLeod, in EPAC08, Genoa, Italy (2008), Vol. 1, pp. 1149-1151.

${ }^{10}$ F. G. Sun, Z. Jiang, and X.-C. Zhang, Appl. Phys. Lett. 73(16), 2233-2235 (1998).

${ }^{11}$ J. Fletcher, Opt. Express 10(24), 1425-1430 (2002).

${ }^{12}$ G. Berden, S. P. Jamison, A. M. MacLeod, W. A. Gillespie, B. Redlich, and A. F. G. van der Meer, Phys. Rev. Lett. 93(11), 114802 (2004).

${ }^{13}$ M. H. Helle, D. F. Gordon, D. Kaganovich, and A. Ting, Phys. Rev. Spec. Top.-Accel. Beams 15(5), 052801 (2012).

${ }^{14}$ R. Huber, A. Brodschelm, F. Tauser, and A. Leitenstorfer, Appl. Phys. Lett. 76(22), 3191-3193 (2000).

${ }^{15}$ E. Matsubara, M. Nagai, and M. Ashida, Appl. Phys. Lett. 101(1), 011105 (2012).

${ }^{16}$ D. J. Kane and R. Trebino, IEEE J. Quantum Electron. 29(2), 571-579 (1993).

${ }^{17}$ R. Trebino, K. W. DeLong, D. N. Fittinghoff, J. N. Sweetser, M. A. Krumbügel, B. A. Richman, and D. J. Kane, Rev. Sci. Instrum. 68(9), 3277-3295 (1997).

${ }^{18} \mathrm{R}$. Trebino, FROG (Springer, US, 2000).

${ }^{19}$ C. Iaconis and I. A. Walmsley, Opt. Lett. 23(10), 792-794 (1998).

${ }^{20}$ E. W. Snedden, D. A. Walsh, and S. P. Jamison, Opt. Express 23(7), 8507-8518 (2015).

${ }^{21}$ G. Gallot and D. Grischkowsky, J. Opt. Soc. Am. B 16(8), 1204-1212 (1999).

${ }^{22}$ S. P. Jamison, A. M. MacLeod, G. Berden, D. A. Jaroszynski, and W. A. Gillespie, Opt. Lett. 31(11), 1753-1755 (2006).

${ }^{23}$ Q. Chen, M. Tani, Z. Jiang, and X. C. Zhang, J. Opt. Soc. Am. B 18(6), 823-831 (2001).

${ }^{24}$ L. Duvillaret, S. Rialland, and J.-L. Coutaz, J. Opt. Soc. Am. B 19(11), 2704-2715 (2002).

${ }^{25}$ S. Casalbuoni, H. Schlarb, B. Schmidt, P. Schmüser, B. Steffen, and A. Winter, Phys. Rev. Spec. Top.-Accel. Beams 11(7), 072802 (2008).

${ }^{26}$ S. P. Jamison, Appl. Phys. B: Lasers Opt. 91(2), 241-247 (2008).

${ }^{27}$ I. N. Ross, P. Matousek, G. H. C. New, and K. Osvay, J. Opt. Soc. Am. B 19(12), 2945-2956 (2002).

${ }^{28}$ P. O'shea, M. Kimmel, X. Gu, and R. Trebino, Opt. Lett. 26(12), 932-934 (2001). 